\title{
IMECE2013- 66872
}

\section{EXPLORATION OF IMPINGING WATER SPRAY HEAT TRANSFER AT SYSTEM PRESSURES NEAR THE TRIPLE POINT}

\author{
Eric L Golliher \\ NASA Glenn Research Center \\ Cleveland, Ohio, USA
}

\author{
Shi-chune Yao \\ Carnegie Mellon University \\ Pittsburgh, Pennsylvania, USA
}

\begin{abstract}
The heat transfer of a water spray impinging upon a surface in a very low pressure environment is of interest to cooling of space vehicles during launch and re-entry, and to industrial processes where flash evaporation occurs. At very low pressure, the process occurs near the triple point of water, and there exists a transient multiphase transport problem of ice, water and water vapor. At the impingement location, there are three heat transfer mechanisms: evaporation, freezing and sublimation. A preliminary heat transfer model was developed to explore the interaction of these mechanisms at the surface and within the spray.
\end{abstract}

Keywords: Multiphase Flow, Spray Cooling, Evaporation, Heat Transfer

\section{INTRODUCTION}

This research is an investigation of heat transfer from a flat surface using water spray cooling at system pressures that are below standard atmospheric pressure. The focus is on those system pressures near the triple point of water. There has been limited research in this area. At the triple point of water, the three phases are in equilibrium: liquid, vapor and solid, at $0.01^{\circ} \mathrm{C}$ and $612 \mathrm{~Pa}$ (4.6 Torr). An understanding of the physical processes of operating below the triple point is needed for thermal control systems of future space missions.

The original interest in this research comes from Space Shuttle Orbiter hardware called the Flash Evaporator System (FES). This hardware cools the Orbiter main coolant loop to approximately $4{ }^{\circ} \mathrm{C}$ during certain mission phases. The desired system pressure and temperature were always above the triple point of water, so that freezing would hopefully never occur. Although the FES has been mostly successful, some past shuttle missions have had anomalies of this hardware [1]. It is thought that unexpected icing caused the issue and that the anomalies of the FES were caused by some blockage or other inability of the passive backpressure device to create the proper system pressure within the FES. If a good understanding of the icing mechanism and heat transfer for a spray evaporative cooling system can be achieved, it is possible that future space vehicles may have the option of using a more efficient spray evaporative cooling system, thereby saving vehicle weight for more payload items.

The Shuttle FES was developed in the late 1970's by Hamilton Standard of Windsor Locks, Connecticut. Since that era, newer published research results may help better understand low system pressure spray cooling process, and the associated anomalies of the shuttle FES.

Most publications in the past have provided heat transfer information for spray cooling at system pressures that are near or above the triple point. Only one publication has provided freezing data far below the triple point, but no heat transfer model. The research described in this paper proposes both a preliminary heat transfer model and freezing data for system pressures far below the triple point. This may allow an understanding of whether this system pressure range is a viable operating point and also, if it is possible to recover from this pressure range should an anomaly occur, whereby the desired system pressure above the triple point cannot be maintained.

Steddum [2] was the first to study water sprays in vacuum conditions. He used a large vacuum chamber in which water was sprayed at an ambient pressure of $2 \times 10^{-5}$ Torr. The pressurization method for the nozzle consisted of a pressurized gas cylinder forcing water through the nozzle. A high speed camera at 4700 frames per second recorded the freezing of the droplets and the velocity. A plate was placed inside the chamber at a distance two to six feet from the spray. The plate was used to determine if the droplets had frozen. If they bounced off the plate, they were assumed frozen. If they stuck to the plate and led to ice accumulation on the plate, the droplets were assumed to have been liquid. Steddum did not measure droplet size, but did reason that there existed a range of droplet sizes in a given 
spray, and that smaller droplets would freeze faster. In the analysis section, he assumed the droplets were on the order of 1000 micron diameter. The freezing times reported by Steddum, for lower pressure sprays, were from $110 \mathrm{~ms}$ to 130 ms. No attempt at resolving the heat transfer of the impacting droplets was made.

In 1979, Grissom [3] studied spray cooling in vacuum conditions. He found the maximum heat flux for a given set of conditions that related to the design of the FES. Grissom studied system pressure slightly below the triple point at 4.59 Torr. His limited data can therefore not be used to evaluate the mechanisms of freezing caused by very low system pressures on the order of 0.01 Torr. Also in 1979, Rizza [4] studied a water spray in vacuum at the triple point, but not below the triple point. He found that splash and stick droplet dynamics did not apply near the triple point, since partial solidification (freezing) of the droplets affected the impact.

In 1985, Yanosy [5] developed a relationship to predict heat transfer for spray cooling in a vacuum. He only considered system pressures above the triple point pressure of water, which is the nominal operating condition of the FES.

\section{NOMENCLATURE}

$\begin{array}{ll}P & \text { Pressure, Pa } \\ T & \text { Temperature, K } \\ x & \text { Heat flow dimension, } \mathrm{m} \\ q & \text { Heat Flow, W } \\ L & \text { Disc thickness, } \mathrm{m} \\ c_{p} & \text { Specific Heat, } \mathrm{J} / \mathrm{kg} \mathrm{K} \\ t & \text { Time, } \mathrm{s}\end{array}$

\section{Greek Symbols}

$\Phi \quad$ Parameter used in Inverse Heat Conduction Method

$\lambda \quad$ Parameter used in Inverse Heat Conduction Method

\section{Subscripts}

system Pertaining to the chamber environment

droplet vapor Conditions determined by the droplet

\section{APPROACH}

This research investigates the mechanism of freezing and heat transfer of an impacting water spray at system pressures below the triple point of water. Experiments were performed to quantify the heat transfer and provide evidence to support a proposed heat transfer mechanism. This data should provide a first understanding of the mechanism, and help outline possible future research. This was accomplished using the analysis technique of Inverse Heat Conduction (IHC). Using the transient temperature response of a small $0.0254 \mathrm{~m}$ diameter, $0.001 \mathrm{~m}$ thick copper disc impacted by a water spray, the IHC method determines the heat flux time history. The characteristics of this transient heat flux curve indicate a possible mechanism for heat transfer and freezing. A small chamber suitable for vacuum levels below the triple point contains a custom water spray nozzle. This nozzle is composed of an automotive fuel injector, whereby the original atomizer is replaced with a very low flowrate swirl nozzle [6]. The spray pulse duration is controlled by a programmable logic controller that can switch the injector solenoid on and off with a pulse duration of as short as 10 milliseconds (ms). A laboratory vacuum pump was used to achieve system pressures as low as 0.01 Torr. The equilibrium water temperature corresponding to this system pressure is about $-58^{\circ} \mathrm{C}$.

The copper disc has two thermocouples mounted on the back side, one near the center and one near the edge. A halogen lamp shining through the vacuum chamber window provides illumination for the video camera and also provides enough heat to warm the copper disc to an initial temperature of up to $45^{\circ} \mathrm{C}$.

\section{EXPERIMENTAL SETUP}

Two type-T thermocouples are attached with epoxy to the back side of a $0.0254 \mathrm{~m}$ diameter, $0.001 \mathrm{~m}$ thick, gold/nickel coated pure copper disc. The epoxy has a thermal conductivity of $1 \mathrm{~W} / \mathrm{mK}$, is suitable for vacuum applications, and is electrically insulating. The copper disc rests on two thin strings of polyimide to eliminate parasitic thermal conduction from the support structure. The thermocouples are recorded and processed with a data acquisition system capable of 6400 measurements per second. The high seed camera settings were 1000 frames per second at 1632 X 1200 pixels. The nozzle pressure is controlled by a manual valve and measured with a Bourdan pressure gauge. The nozzle is a low flow swirl buttontype nozzle normally used for fuel vaporization in jet engines. This nozzle is attached to a modified solenoid fuel injector with the blue-colored vacuum-compatible epoxy as shown in Fig. 1.

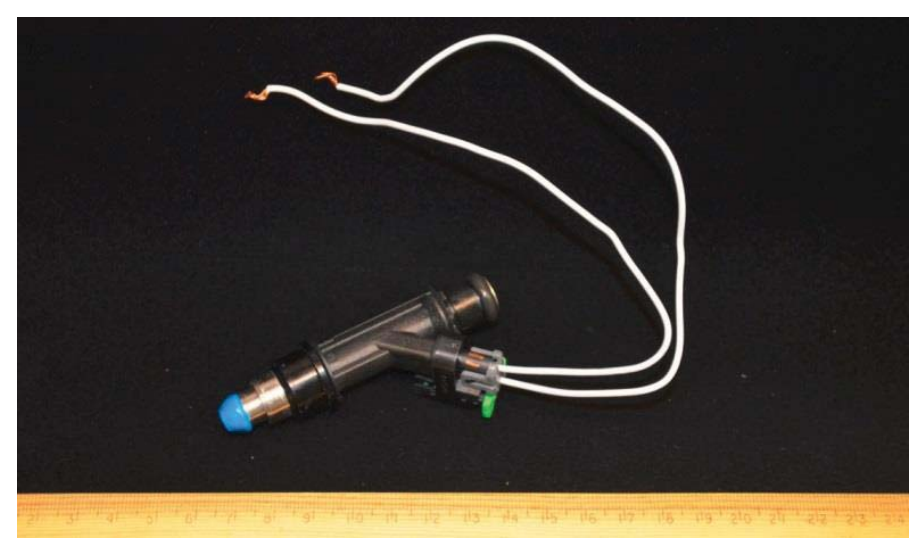

FIGURE 1. NOZZLE AND SOLENOID INJECTOR

A standard halogen lamp is located outside the chamber but is directed to illuminate the spray at a $150^{\circ}$ angle from the viewing window, as can be seen in Figure 2. This angle appears to be ideal for viewing droplets that will refract the light towards the camera. 


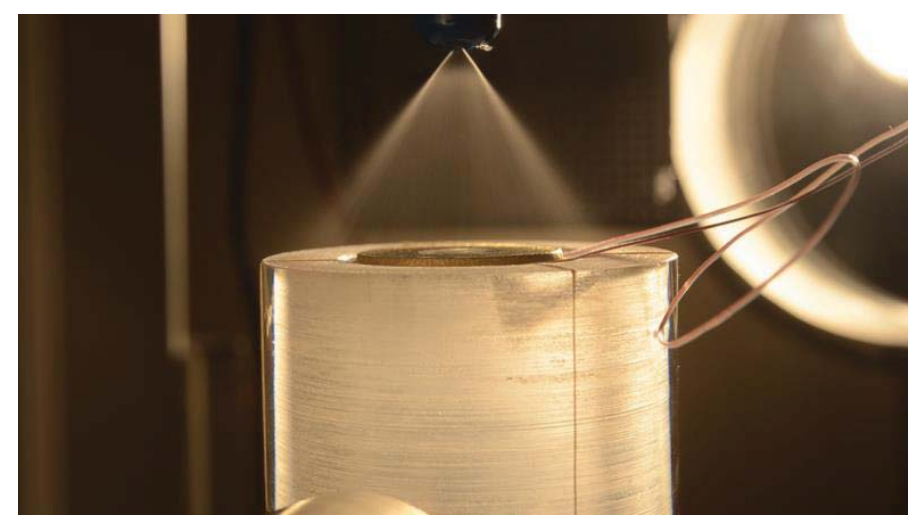

FIGURE 2. COPPER DISC SUSPENDED ON POLYIMIDE THREAD

Figures 3 and 4 show the experimental setup. The pressure sensor is a capacitance manometer with a range of 0 to 100 Torr. The water is delivered to the injector by a plunger-type piston, whereby the air side of the piston is pressurized by a facility regulated air system, and the water side of the piston is manually filled with distilled water. By separating the water from the pressurized air, there is the capability of degassing the water before filling, and then assuring the water remains degassed during the experiment. The solenoid controller is programmed to allow variable open and close times for the nozzle spray. The vacuum pump is capable of handling small amounts of water vapor such as anticipated in this experiment. For low system pressures, the spray cone angle increases from that observed at higher system pressures. Therefore, the nozzle to plate distance must be changed accordingly to ensure that the spray impact circle matches the disc area.

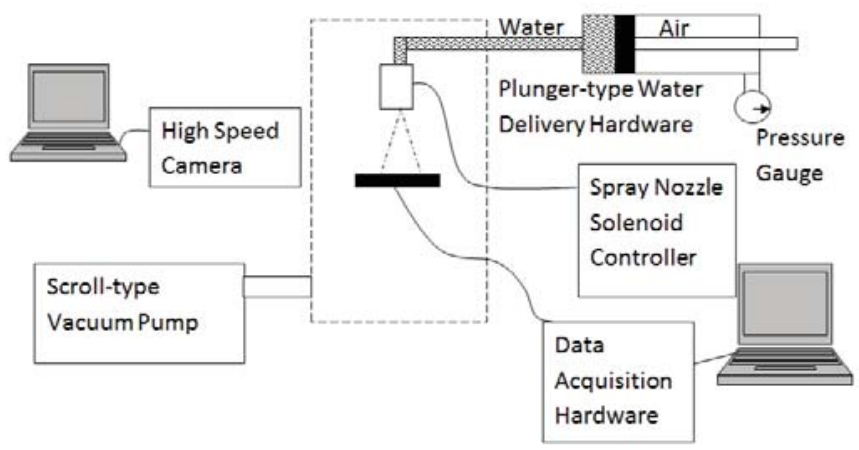

FIGURE 3. EXPERIMENTAL SETUP

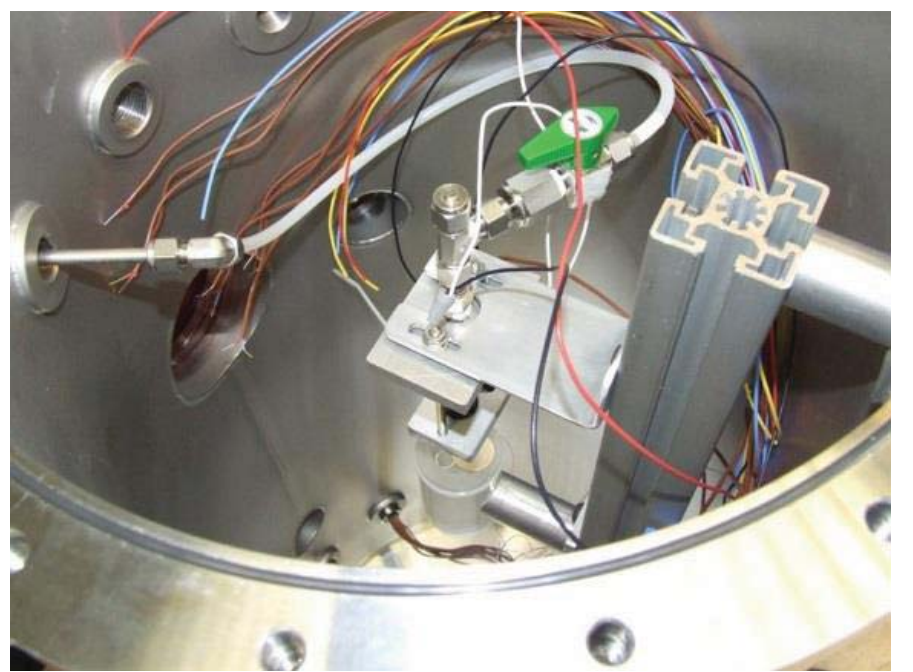

FIGURE 4. VIEW SHOWING MOUNTING STRUCTURE OF NOZZLE

The chamber volume is $0.08367 \mathrm{~m}^{3}$ with the following feedthroughs: copper/constantan for thermocouples, power, pressure measurement, vacuum pump duct, and water entrance.

\section{RESULTS AND DISCUSSION}

In this section, the data observations are related to a thermal analysis, after which the uncertainty in the data and a possible model are discussed.

\section{Data Observations}

Data of temperature versus time has been acquired to illustrate the capabilities of the experimental hardware and illustrate the proposed data analysis process, as shown in Figure 5. The data acquisition system produces 6400 data points per second. The random error in the data causes the data to fluctuate by about $2{ }^{\circ} \mathrm{C}$. The random error is removed and the data smoothed via curvefit software.

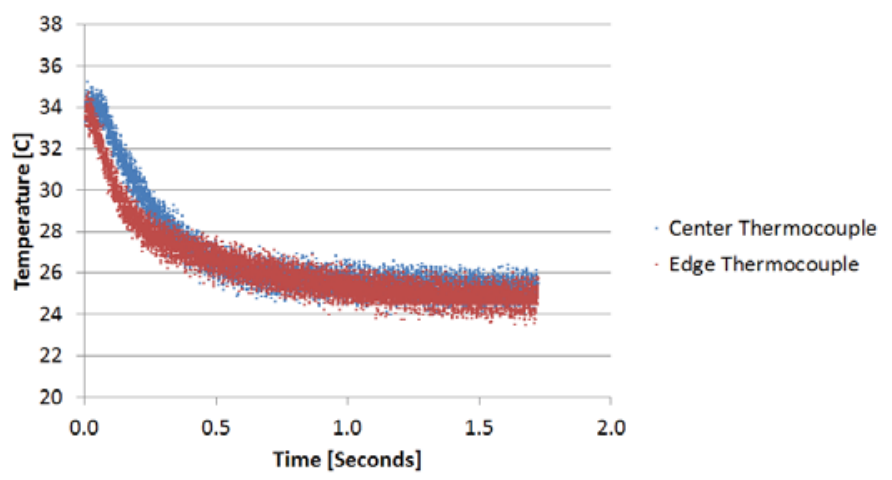

FIGURE 5. TEMPERATURE VERSUS TIME HISTORY 
The spray valve is opened by a $14 \mathrm{~V}$ DC signal sent to the solenoid. The time of this signal is recorded along with the temperature history of both thermocouples. In this way, the start of the spray is known.

There is some small error introduced by assuming the solenoid opening is the start of the spray impact, but it is negligible when compared to the total response time of the 50 ms spray event. Since the spray droplet velocity is approximately $5.2 \mathrm{~m} / \mathrm{s}$ and the nozzle to plate distance is 1.6 $\mathrm{cm}$, the time of flight for the droplets to travel from the nozzle and impact the disc is about $3 \mathrm{~ms}$. Further, high speed video has shown the time for the spray to fully develop is about $3 \mathrm{~ms}$. Also, the observed timescale for the copper disc temperature response is about 1.7 seconds. Therefore, the spray startup and time of flight lag are both negligible compared to the timescale of the disc temperature response. Even though the initial time is set to that of the opening of the solenoid, this introduces little error in the inverse heat conduction analysis.

The spray itself was observed to increase in cone angle as the system pressure decreased. This is consistent with published literature for studies of sprays at system pressures above and below atmospheric levels. From the work of DeCorso and Kemeny [7], it has been observed that for pressures higher than standard atmospheric, such as in diesel combustion, the spray cone angle is smaller. The reason is that the high velocity within the spray cone leads to lower pressure there, as compared to the stagnant ambient pressure air. The ambient air is pulled into the cone, entraining the droplets, producing drag on the droplets as the ambient air moves toward the center of the spray, and leading to a smaller effective cone angle. For pressures in the range of 10 to $800 \mathrm{kPa}$, DeCorso and Kemeny experimentally report the relationship of cone angle to system pressure in Eq. (1).

$$
\text { Spray Cone Angle } \quad \propto \frac{1}{P_{\text {system }}{ }^{1.6}}
$$

Since this was measured at systems pressures no lower than $10 \mathrm{kPa}$, the question remains as to whether this relationship and reasoning for spray angle change with system pressures are valid for system pressures far below $10 \mathrm{kPa}$.

To answer the question of spray angle versus system pressure, the spray angles for various system pressures below the triple point were measured graphically. Figures 7 through 10 show the variation of spray angle with system pressure.

The spray angle data taken from video data is presented in Fig. 7 through Fig. 10 and plotted in Fig. 6. The variation in spray angle with system pressure appears to be linear for system pressures in the continuum regime.

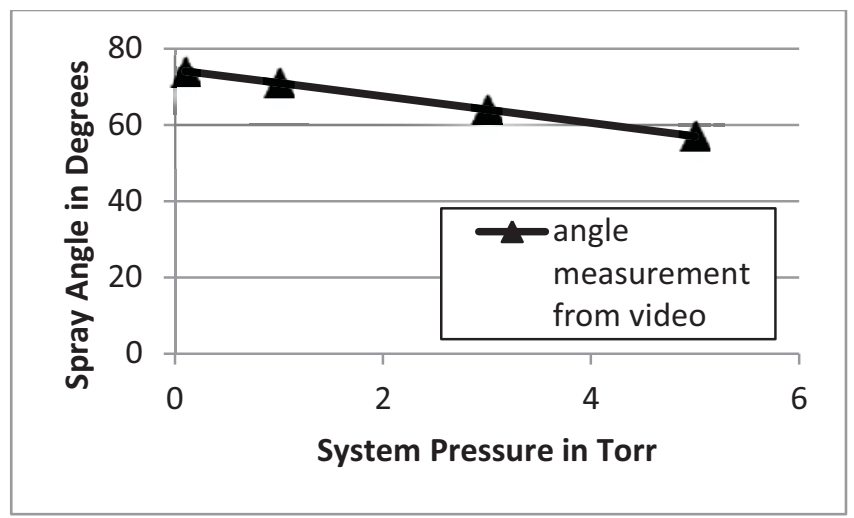

FIGURE 6. SPRAY ANGLE DATA

As the pressure decreases from 5 Torr to 0.1 Torr, the Knudsen number (the mean free path compared to the length scale) ranges from about one to 26 , and indicates non-continuum flow. With negligible ambient air, the reasoning of DeCorso and Kemeny does not apply in this pressure range. In non-continuum flow, Carey [8] shows a linear relationship between evaporation flux and system pressure in Equation (2).

$$
\text { Evaporation } \propto\left(\frac{P_{\text {droplet vapor }}}{\sqrt{T} \text { droplet vapor }}-\frac{P_{\text {system }}}{\sqrt{T}_{\text {system }}}\right)
$$

The data plotted in Figure 6 also shows a linear relationship with system pressure in Equation (3).

$$
\begin{aligned}
& \text { Spray Cone Angle } \propto-P_{\text {system }} \\
& \text { for } P_{\text {system }}<5 \text { Torr }(667 \mathrm{~Pa})
\end{aligned}
$$

As the system pressure decreases, the droplet evaporation increases; that is, the amount of vapor molecules blowing outward from the spray core increases. The pressure inside the core is greater than outside. As more water vapor blows outward, it entrains and pushes the droplets away from the spray core. The temperature and vapor pressure of the droplets within the spray remains the same in all cases, but the outside system pressure decreases. Thus, a non-continuum vapor flow model of water vapor blowing away from the spray core may explain the linear trend in data for this limited range of system pressures. 


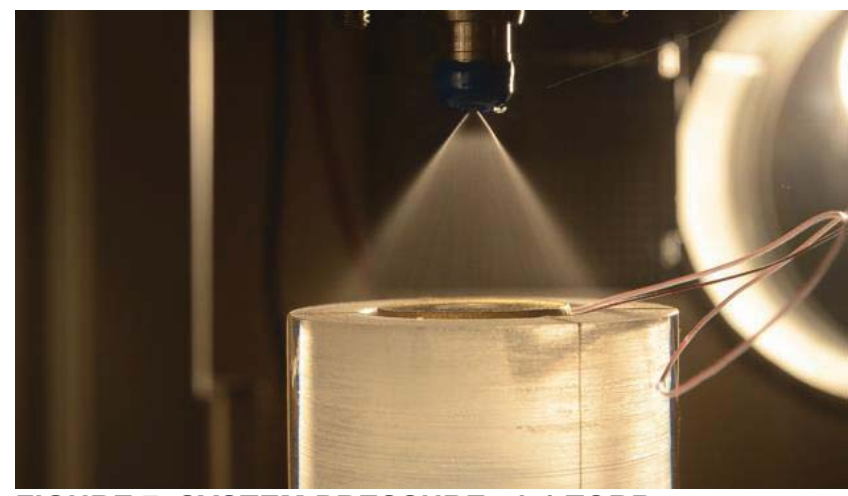

FIGURE 7. SYSTEM PRESSURE $=0.1$ TORR

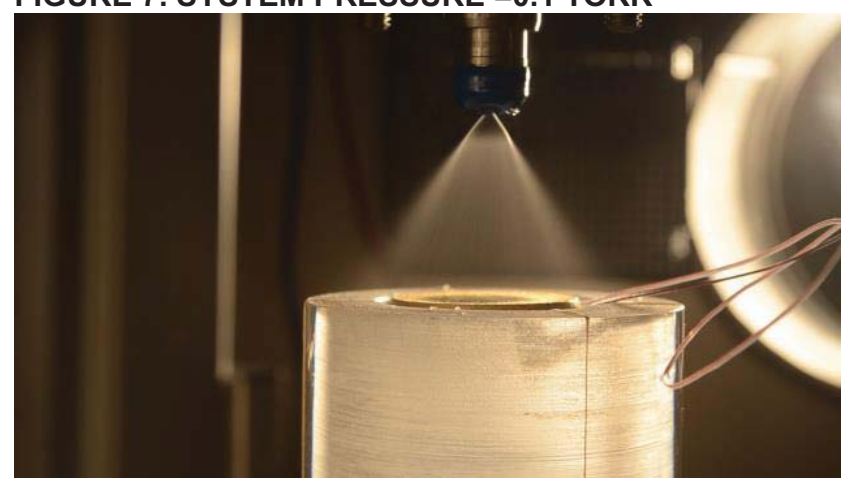

FIGURE 8. SYSTEM PRESSURE = 1 TORR

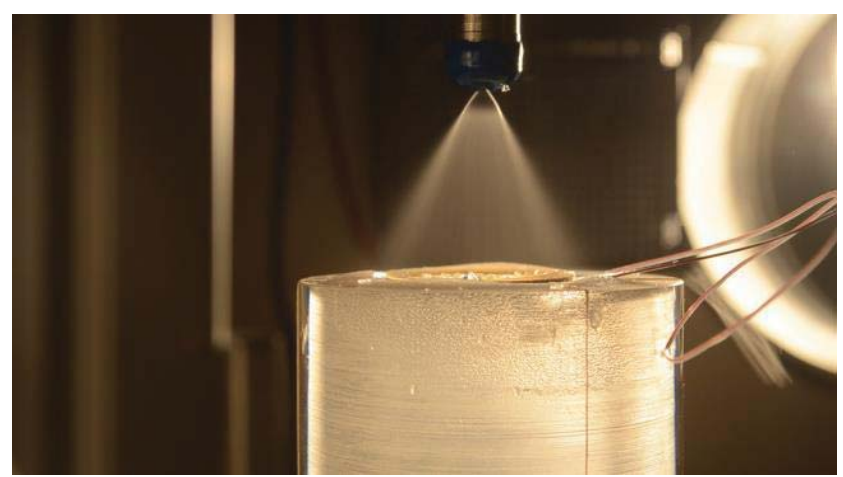

FIGURE 9. SYSTEM PRESSURE = 3 TORR

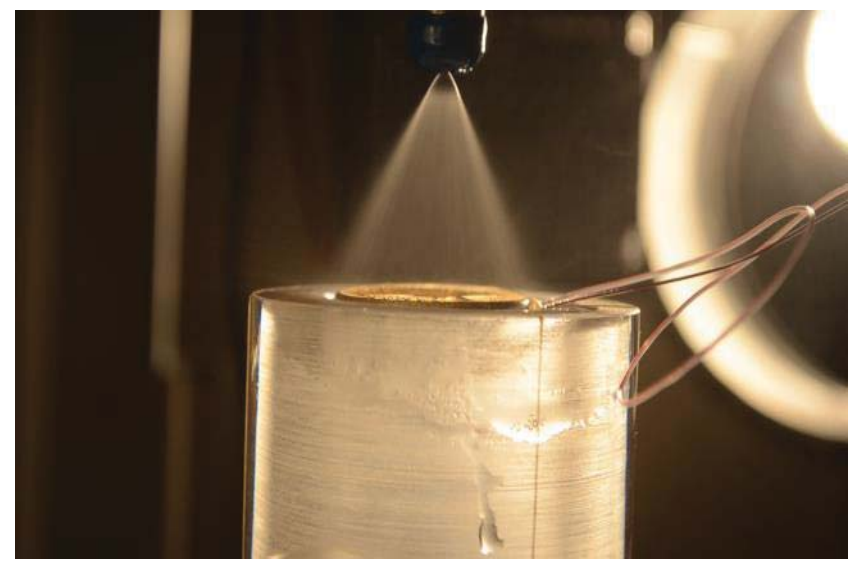

FIGURE 10. SYSTEM PRESSURE = 5 TORR
During a $50 \mathrm{~ms}$ spray pulse, the system pressure increases from 0.01 Torr to 0.26 Torr due to the introduction of water into the chamber. This follows an ideal gas law relation which considers the water vapor as a gas at room temperature. Thus, the equilibrium saturation temperature corresponding to this pressure increase is $-58{ }^{\circ} \mathrm{C}$ to $-31^{\circ} \mathrm{C}$ during a $50 \mathrm{~ms}$ pulse.

When the water pulse occurs, mass is introduced to the closed chamber, and the pressure rises. Using the ideal gas law, an estimate of chamber pressure rise can be made by considering the change in specific volume of water vapor, and by assuming that, at 0.01 Torr initial pressure, the amount of air in the chamber is negligible. Results show that after one $50 \mathrm{~ms}$ pulse, the chamber pressure should be 0.26 Torr. Experimental results confirm this prediction.

Successive $50 \mathrm{~ms}$ pulses cause an increase in chamber pressure and a corresponding decrease in disc temperature, with ice buildup on the disc. At some point the equilibrium pressure and temperature of ice is reached whereby no additional evaporative cooling takes place.

Two cases are possible: 1) the vacuum pump is off and the chamber is closed, and 2) the vacuum pump remains on.

Results for case 1 are shown in Figure 11. After 6 successive pulses of $50 \mathrm{~ms}$, ice/vapor equilibrium is reached and additional disc cooling stops. Pulsing is continued and the incoming water is frozen and added to the ice already resident on the disc. The disc temperature and chamber pressure slowly rise in equilibrium. The equilibrium temperature and pressure after 6 pulses is measured at about- $14{ }^{\circ} \mathrm{C}$ and 1.41 Torr. This compares well with the most recent accepted relations between vapor pressure over ice to temperature, the Arden Buck equation of 1996 [9], which predicts 1.36 Torr.

$$
P_{\text {system }}=611.15 e^{\left(\frac{\left(23.036-\frac{T}{333.7}\right) T}{297.82+T}\right)}
$$

The Arden Buck equation relates the ice temperature to the system pressure. For this equation, the temperature is in the units of Celsius and the system pressure in Torr. 

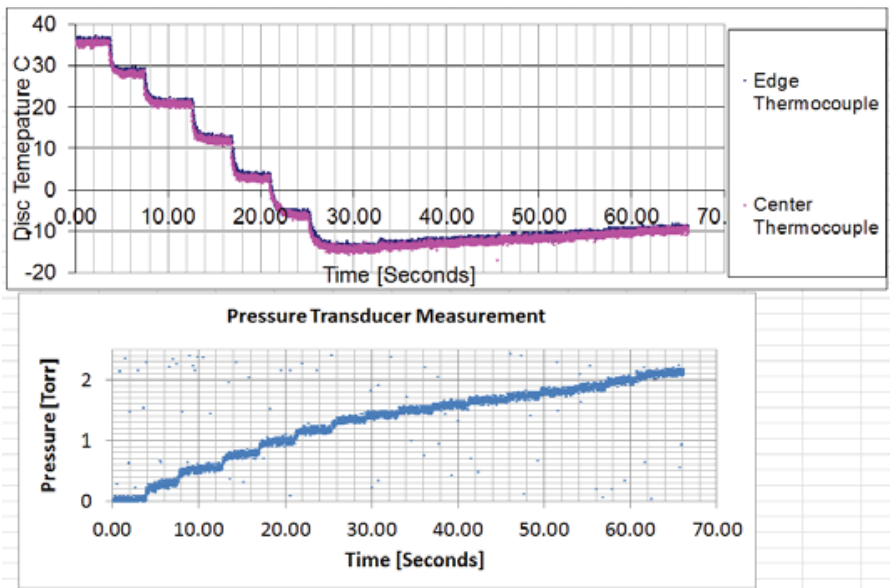

\section{FIGURE 11. DISC TEMPERATURE AND CHAMBER PRESSURE WHEN ICE IS RESIDENT ON THE DISC ( CASE 1 PUMP OFF)}

Results for case 2 are shown in Figure 13. The equilibrium temperature and pressure after multiple pulses is measured as $-23{ }^{\circ} \mathrm{C}$ and 0.62 Torr. This value compares well with the Arden Buck equation plotted in Figure 12. At this point of equilibrium, the vacuum pump is removing water vapor at the rate it is being introduced to the chamber by the multiple $50 \mathrm{~ms}$ pulses. After the pulsing is stopped at about 85 seconds, the vacuum pump continues to remove mass from the chamber and the temperature continues to decreases as expected

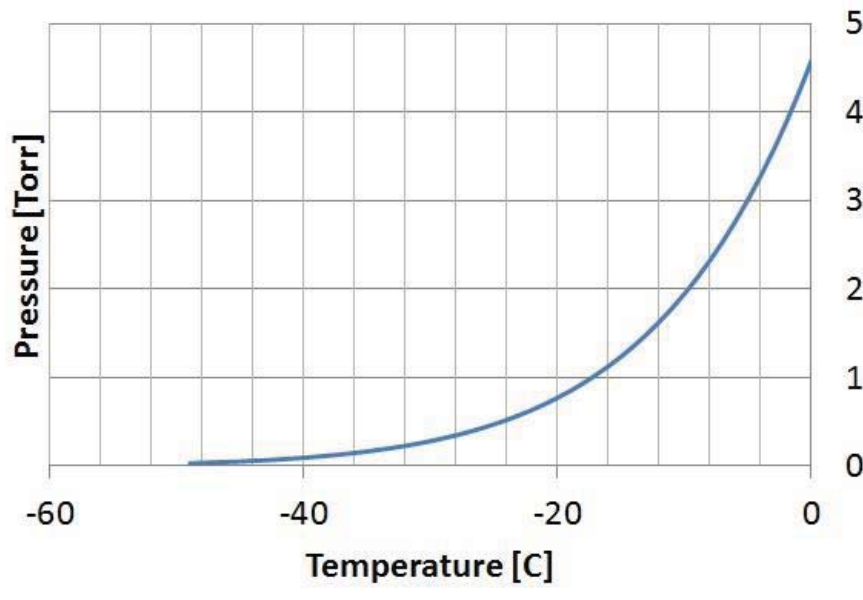

FIGURE 12. ARDEN BUCK RELATION FOR VAPOR PRESSURE OVER ICE
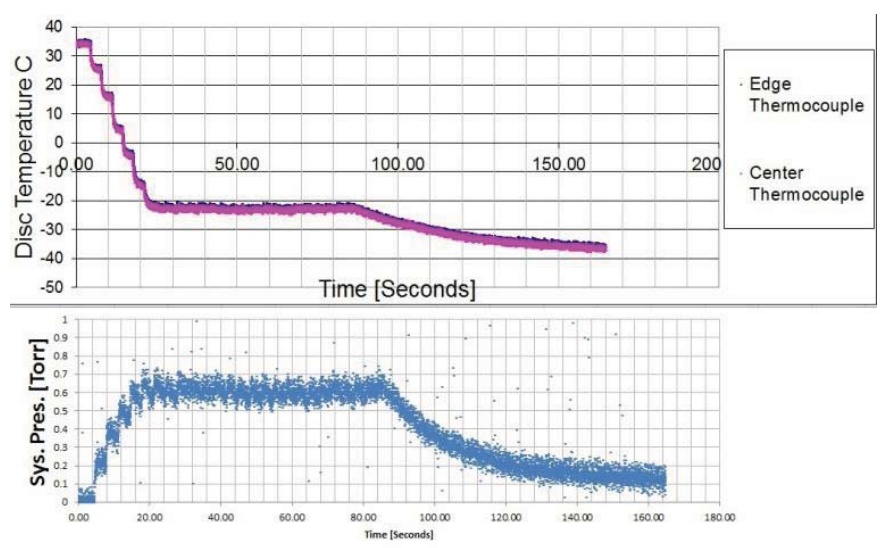

FIGURE 13. DISC TEMPERATURE AND CHAMBER PRESSURE WHEN ICE IS RESIDENT ON THE DISC (CASE 2 PUMP ON)

\section{Thermal Analysis}

There have been two analyses conducted for assisting the understanding of the experiments. First, the temperature response of the copper disc (cooling) caused by the impacting spray. Residual water remains on the disc even after the spray cutoff, and continues to cool the disc until completely evaporated. Second, the droplet temperature leaving the nozzle is at room temperature, but decreases during the flight from nozzle to disc. The sensible heat of the droplets supplies the latent heat of evaporation. Since, at vacuum conditions, there is no surrounding medium to supply heat to the droplet, all the evaporative latent heat must be supplied by the temperature change of the remaining liquid in the droplet.

After the spray pulse stops, and the disc continues to cool due to the residual water evaporation, it is possible that the top surface forms an ice layer and the freeze front moves towards the disc. Heat transfer begins to decrease because ice slows any convective or pool boiling component inside the liquid drop. The water layer completely freezes, then sublimates, and then completely disappears. The ice/vacuum interface could be very uneven, but it assumed flat for now in this analysis.

A one dimensional model of the copper disc characterizes axial heat transfer through the disc. This analysis assumes a spatially uniform heat loss on the water-impinging (top) side of the disc at $\mathrm{x}=0$. Because this research focuses on system pressures below the triple point, the boundary at $\mathrm{x}=\mathrm{L}$ can be assumed insulated, as convective losses are negligible. 


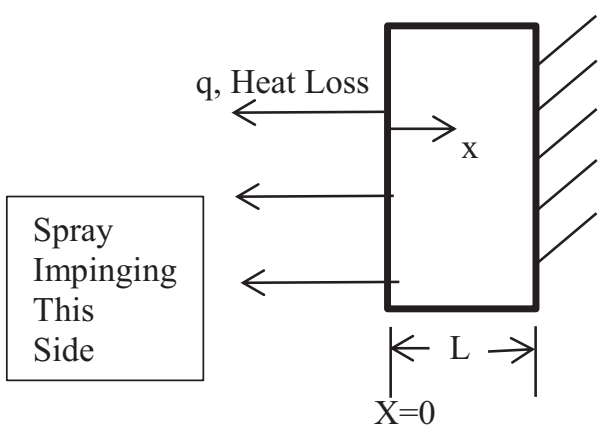

FIGURE 14. COPPER DISC MODEL

The governing equation for this problem is:

$$
\begin{gathered}
\frac{\partial^{2} T}{\partial x^{2}}=\frac{1}{\alpha} \frac{\partial T}{\partial t} \\
T(x, 0)=T_{o} \\
\frac{\partial T}{\partial x}=0 \text { at } x=L \\
\text { and } \\
T(L, t)=\text { known from experimental data } \\
q(0, t) \text { and } T(0, t) \text { are desired }
\end{gathered}
$$

Equations (4) through (6) have an analytical solution for a constant heat flux, but not for a varying heat flux. The method of inverse heat conduction is used to find the transient heat flux on the left of the disc, at $\mathrm{x}=0$, given the transient temperature profile provided by a thermocouple attached to the right of the disc at $\mathrm{x}=\mathrm{L}$. As outlined in Beck [11], the problem formulates as the matrix form of Duhamel's theorem. Duhamel's theorem allows the estimation of temperature within a slab for a varying heat load, by first forming an auxiliary problem whereby the heat load is constant. A parameter, $\varphi$, shown in Equation (7), is defined that essentially linearizes the change in temperature with a change in heat flux.

$$
\phi=\frac{\partial T(x=L, t)}{\partial q(x=0)}
$$

Then, for the inverse heat conduction problem, when one divides the known temperature time history by $\varphi$, one can estimate the unknown heat flux time history. In matrix form, integrating the change in $\varphi$ with time, and multiplying by the heat flux, the method returns an estimate of the change in temperature at $\mathrm{x}=0$ for a chosen discrete set of time increments as shown in Equation (8).

$$
T(x, t)=\int_{0}^{t} q(\lambda) \frac{\partial \phi(x, t-\lambda)}{\partial t} d \lambda
$$

Taking the discrete set of time steps as 10 , for convenience, and arranging this integral in series of ten discrete time steps, allows for the creation of what is called the pulse sensitivity coefficient matrix, $\mathbf{X}$.

The arbitrary choice of 10 here is to illustrate the method. This number may be optimized by trial and error. If the number of increments is too large, the method is unstable. If the number of increments is too small, information may be missed.

Then, Equation (9) defines the matrices to be solved.

$$
\mathbf{T}=\mathbf{X} \mathbf{q}
$$

Since the time history of temperature, $\mathbf{T}$ and the pulse sensitivity coefficient matrix, $\mathbf{X}$, are known, for the transient heat flux, q, can be determined.

In Beck [10], much of the text is devoted to solution methods for these types of matrices and for many different possibilities of data and variations in the physical problem. However, some modern, commercially-available math software packages can solve this problem easily. This yields a set of 10 discrete values of heat flux as a function of time.

The inverse heat conduction method, described above, produces discrete points as an estimate of transient heat flux at $\mathrm{x}=0$, based on the temperature that is available at $\mathrm{x}=\mathrm{L}$. The plot in Figure 15 shows heat flux in Watts per meter squared versus time in seconds. A point at the origin at time zero was added manually for completion. In reality, the IHC method cannot predict the value of heat flux at very small times values near time $=0$. The spray duration is 50 milliseconds. The end time is 172 milliseconds. Each point represents the midpoint of the time increment, e.g., the first midpoint is at 86 milliseconds. Since each point represents the midpoint of the time increment, the final time of the temperature data is 1.72 seconds, but the final midpoint of the IHT heat flux data is at 1.64 seconds. 


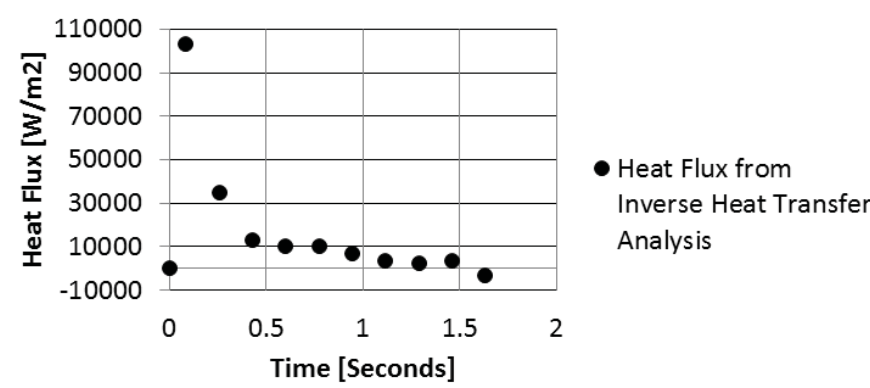

\section{FIGURE 15. HEAT FLUX HISTORY DERIVED FROM INVERSE HEAT CONDUCTION METHOD}

\section{Uncertainty Analysis}

The error in heat flux prediction is principally due to errors in the change in temperature with time for intervals on the order of 1 second. The uncertainty of the thermocouple measurement is estimated at $+/-1{ }^{\circ} \mathrm{C}$, based on the range of temperatures produced by random noise. There are really two major sources of possible error in the thermocouple measurement: 1) the data acquisition system reading that is different from the true temperature, and 2) high frequency random error produced by unknown electrical noise sources. For a transient temperature measurement spanning less than one second, it is reasonable to assume the difference between the true temperature and the reading is constant, and does not affect the slope of the temperature change with time. Therefore, the only reasonable error estimate of the temperature change with time is due to the high frequency error that fluctuates by about $+/-1{ }^{\circ} \mathrm{C}$. Since the $+/-1{ }^{\circ} \mathrm{C}$ temperature range appears to include most or all of the random noise temperature measurements, we can assume this is a $3 \sigma$ value. If this is true, then one standard deviation is $0.3{ }^{\circ} \mathrm{C}$ above or below the true value. Then, in order to estimate error in heat flux, we can assume that the slope of the temperature measurement with time increases by $0.6{ }^{\circ} \mathrm{C}$ per measurement time interval, since the first measurement might be too low by one standard deviation and the second might be too high. Since the temperature profile is divided into 10 points, the time interval is 0.174 seconds for the case used in this paper and shown in Fig. 15. Further, we can assume a straightforward lumped parameter analysis of the copper disc and relate heat flux to the time rate of temperature change. For the copper disc:

$$
L \rho c_{p} \frac{d T}{d t}=q
$$

In this linear relationship of temperature change with time and heat flux, the $\rho, c_{p}$, are the values for pure copper. If $0.6{ }^{\circ} \mathrm{C}$ and
0.174 seconds are used for the temperature change and time interval, then the resulting value of change in heat flux is 11,900 $\mathrm{W} / \mathrm{m}^{2}$.

The additional uncertainty in the Data Acquisition System (DAQ) electronics resolution appears negligible, since this DAQ uses a 16-bit analog-to-digital converter, the Texas Instruments ADS7805. The resolution of this DAQ is approximately 0.0005 ${ }^{\circ} \mathrm{C}$, which was obtained from inspection of the data.

The error in system pressure measurement appears to be small. The method to check this was as follows: the pressure and disc temperature were recorded with ice resident on the disc after several repeated spray pulses. Additional spray pulses produced no further temperature reduction, which indicated that the system vapor pressure and temperature in the chamber were at equilibrium. The temperature and pressure were checked with the empirical equation provided by Arden Buck for water vapor pressure over ice. For disc temperatures near $-20{ }^{\circ} \mathrm{C}$, the pressure difference between measured and predicted was $4 \%$.

\section{Discussion}

During the spray pulse, the spray is continually supplying the disc with additional water. Since the water is well mixed because of the impacting spray droplets, the water resident on the disc has an essentially uniform temperature. The enthalpy of the impinging droplets is much greater than the heat loss due to evaporation. Therefore, the water temperature does not change during the spray and remains the same temperature as that of the incoming droplets $\left(\sim 12{ }^{\circ} \mathrm{C}\right)$ for the duration of the spray One interesting point is the discussion by Bewig [11], that the contact angle between the water and gold-coated copper disc greatly changes the heat transfer in the boiling regime. Since the copper disc surface was wiped with alcohol to clean it before taking data, an organic monolayer was established. Since the surface property determines the contact angle, the procedure was consistent for each test. According to Bewig [11] even a small amount of organic contaminant, will greatly affect contact angle. Ensuring a repeatable contact angle is important to correlating a model across different data sets. By inspection, the water/gold-coated copper disc contact angle is about 27 degrees at atmospheric pressure.

Considering all of the above, the heat transfer mechanism might progress as follows: vapor bubbles develop near the surface and lead initially to high heat transfer via vaporization. Then, as the freeze front advances downward, toward the surface, it slows any further vaporization. The contact area between the ice and the disc is reduced to the vacuum voids formed during freezing, as shown in Figure 16. After that, sublimation of the remaining ice continues evaporation, but at a low rate. Finally, heat transfer ends when the ice has sublimated away. 


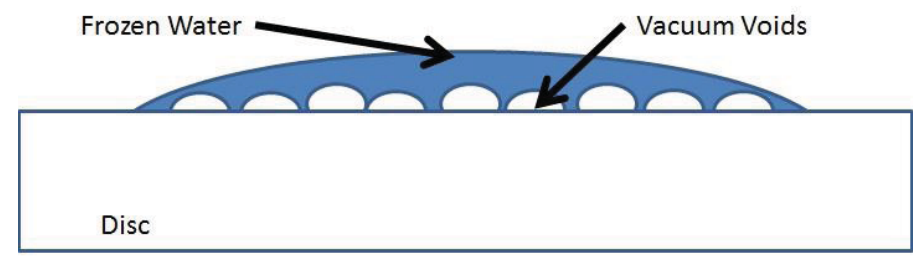

FIGURE 16. CONCEPTUAL ILLUSTRATION OF POSSIBLE MODEL OF ICE FORMATION

The tests and data analyses are ongoing. Details of these new investigations will be reported in the near future. It is possible that evidence of specific heat transfer mechanisms will be available upon completion of a data set that includes variation of system pressure, nozzle pressure, nozzle-to-plate spacing, pulse duration, and pulse frequency.

\section{CONCLUSION}

This testing and analysis of a water spray at system pressures near the triple point of water have resulted in experimental knowledge of spray characteristics such as spray angle and transient heat transfer characteristics. The spray angle appears to increase linearly as system pressure decreases, for system pressures near the triple point of water. Also, the highest heat flux for spray cooling at these system pressures apparently exists just shortly after the initial spray pulse ends. The heat flux decreases rapidly after this.

Although the possibility of using an advanced spray cooling system in future space vehicles is dependent on many additional considerations outside the scope of this paper, it is hoped that understanding of the fundamental engineering heat transfer mechanisms will provide confidence that such systems are viable candidates, and should be developed further.

\section{ACKNOWLEDGMENTS}

The authors would to thank the work of Rex Harvey at Parker Hannifin in Mentor, Ohio, for providing the nozzle.

\section{REFERENCES}

[1] O'Conner, E., Zampiceni, J., Cerna, N., and Fuller, M., 1997, "Orbiter Flash Evaporator: Flight Experience and Improvements," SAE paper No. 972262.

[2] Steddum, R., 1971, "Characteristics of Water Sprays under Vacuum Conditions," Ph.D. thesis, Louisiana State University, Baton Rouge.

[3] Grissom, W. M., 1979, "A Fundamental Study of Spray Evaporative Cooling," Master's Thesis, Rice University, Houston.

[4] Rizza, J., 1979, "Triple Point Pressure Evaporation of Impacted Droplets," AIAA Journal of Spacecraft, 16(4), pp. 210-213.

[5] Yanosy, J., 1985, "Water Spray Cooling in a Vacuum," Ph.D. thesis, University of Connecticut,

[6] Simmons, H. C., and Harvey, R. J., 1999, "Spray Nozzle and Method of Manufacturing Same", Patent\# 5951882
[7] Corso, S. M. D., and Kemeny, G. A., 1957, "Effect of Ambient and Fuel Pressure on Nozzle Spray Angle,"

Transactions of the ASME, 79(3), pp. 607-615.

[8] Carey, V., 1992, Liquid-Vapor Phase-Change Phenomena, Series in Chemical and Mechanical Engineering, Taylor \& Francis,

[9] Buck, A., 1996, "Model Cr-1a Hygrometer Operating Manual,"

[10] Beck, J., Blackwell, B., and Charles St. Clair, J., 1985, Inverse Heat Conduction Ill-Posed Problems, Wiley

Interscience,

[11] Bewig, K. W., and Zisman, W. A., 1965, "The Wetting of Gold and Platinum by Water," Journal of Physical Chemistry, 69(12), pp. 4238-4242. 
